\title{
Fulminant encephalitis as a sole manifestation of COVID-19
}

\author{
Masoud Etemadifar ${ }^{1} \cdot$ Mehri Salari $^{2,3}$ (1) $\cdot$ Aditya Ashok Murgai $^{4} \cdot$ Somayeh Hajiahmadi ${ }^{5}$
}

Received: 6 August 2020 / Accepted: 4 September 2020 / Published online: 8 September 2020

(C) Fondazione Società Italiana di Neurologia 2020

\begin{abstract}
Novel coronavirus (SARS-CoV-2) occurred in December 2019 in Wuhan, China, and has become a global health emergency. Coronavirus primarily is a respiratory virus, but it has been detected in the brain and cerebrospinal fluid of infected individuals. The present report describes a case of fulminant encephalitis in a patient affected by COVID- 19 .
\end{abstract}

Keywords COVID-19 $\cdot$ Encephalitis $\cdot$ Fulminant $\cdot$ Sole presentation

\section{Introduction}

Novel coronavirus (SARS-CoV-2) occurred in December 2019 in Wuhan, China, and has become a global health emergency [1]. Coronavirus primarily is a respiratory virus, but it has been detected in the brain and cerebrospinal fluid of infected individuals [2]. Neurological manifestations can be mild resulting in headache and agitation or more severe causing seizures, encephalitis, stroke, and encephalomyelitis [2]. Neurological and psychiatric consequences of COVID-19 are likely multifactorial due to infection of brain, immunological response, and procoagulant state or due social isolation [3].

Mehri Salari

Mehri.salari@gmail.com

Masoud Etemadifar

Etemadifar.1963@gmail.com

Aditya Ashok Murgai

Murgai03@gmail.com

Somayeh Hajiahmadi

sohajiahmadi@gmail.com

1 Department of Functional Neurosurgery Medical School, Isfahan University of Medical Science, Isfahan, Iran

2 Functional Neurosurgery Research Center, Shohada Tajrish Neurosurgical Center of Excellence, Shahid Beheshti University of Medical Sciences, Tehran, Iran

3 Department of neurology, Shohada-e-Tajrish Hospital, Tehran 1989934148, Iran

4 Zydus hospital, Ahmedabad, India

5 Department of Radiology, Isfahan University of Medical Science, Isfahan, Iran
The present report describes a case of fulminant encephalitis in a patient affected by COVID-19.

\section{Case presentation}

A 51-year-old male, an emergency medicine specialist, presented to the emergency department with 3 days history of headaches and drowsiness. His headaches were episodic, bilateral, and associated with nausea and vomiting. He progressively became drowsy and was brought in the emergency department. He denied history of fever but has dry cough for a week. His past medical history revealed hypothyroidism and migraine. He also gave history of intubating a COVID-19 patient a week back.

His examination revealed conjunctivitis, blood pressure of $90 / 50 \mathrm{mmHg}$, heart rate of 100 beats per minute, oxygen saturation of $98 \%$, and body temperature of $37.6^{\circ} \mathrm{C}$. He was drowsy; following commands, there was no neck stiffness or focal neurological deficit. Electrocardiogram and echocardiography were normal. In the emergency room he had an episode of generalized tonic-clonic seizure followed by a cardiac arrest. He was successfully resuscitated, intubated, and shifted to ICU for further management. His repeated neurological examination showed absence of brain stem reflexes, and his vital parameters were stable.

Blood investigations showed leukocytosis $(14,200 / \mathrm{mm} 3)$ with lymphopenia (11.4\%), elevated D-dimer (1444), prothrombin time (PT), the activated partial thromboplastin time (aPTT), fibrinogen level, red blood cell $\left(4.93 \mathrm{mil} / \mathrm{mm}^{3}\right)$, platelet $\left(201,000 / \mathrm{mm}^{3}\right)$, renal function test, and liver function tests were normal. Brain CT scan showed generalized brain edema 


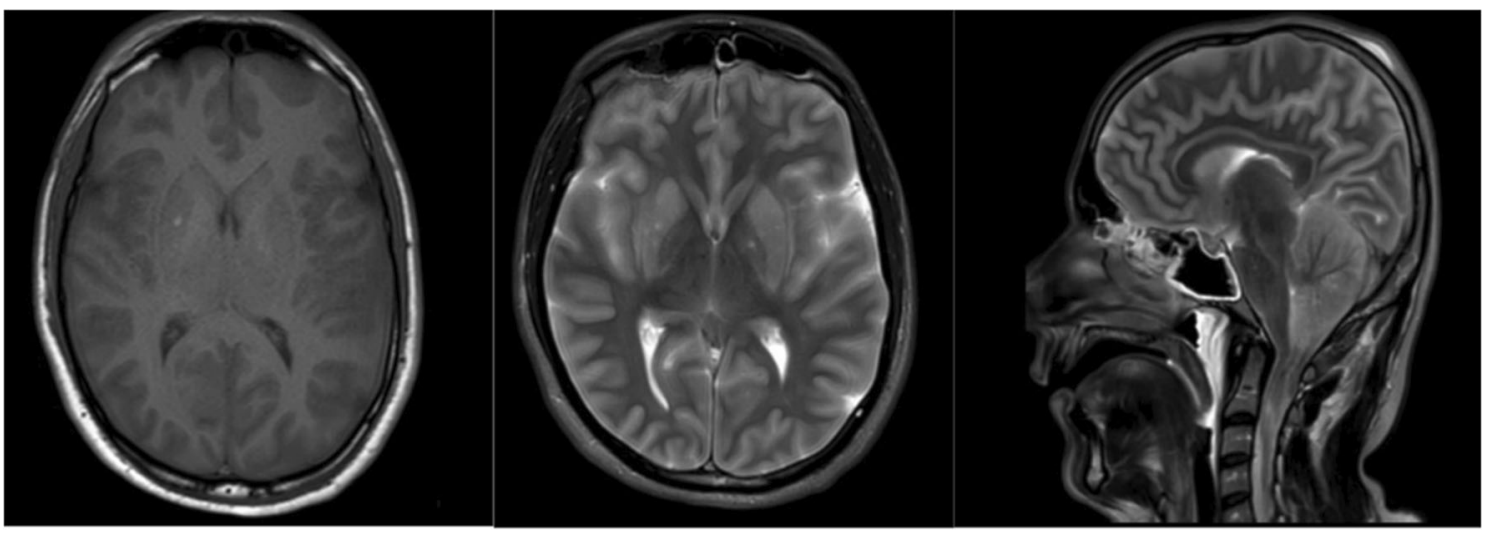

Fig. 1 Axial T1-weighted MR image foci of T1-shortening in the lentiform nuclei are secondary to hemorrhage, axial T2-weighted MR image shows hyperintensity of bilateral frontotemporoparietal cortices

and signs of brain herniation. Chest CT scan was unremarkable. Because of the history of a close contact with a COVID-19 patient, a nasopharyngeal swab was performed which showed a positive result for SARS-CoV-2 on real-time reverse transcriptase polymerase chain reaction assay. He was treated with Hydroxychloroquine (200 mg twice daily), Lopinavir/Ritonavir (400/110 mg twice daily), IV Acyclovir $750 \mathrm{mg} /$ thrice daily, antiepileptic medications, and IV Dexamethasone $4 \mathrm{mg}$ four times a day.

Day two neurological examination did not show any improvement. Brain MRI scan done on day two showed generalized brain edema, downward herniation of cerebellar tonsils and brain stem, and FLAIR hyperintensities in bilateral cerebral cortices and corpus striatum. (Fig. 1) On day three of admission, he developed hypotension and deranged renal functions requiring inotropic support and hemodialysis. Despite all the supportive measures, he died on day three.

\section{Discussion}

We here describe a case of COVID-19 with severe encephalitis, without severe respiratory involvement. Our patient presented with a history of headache, vomiting, altered sensorium, and one episode of generalized seizure, suggesting the diagnosis of meningoencephalitis. His brain imaging showed hyperintensities in bilateral cortices and basal ganglia without diffusion restriction. Brain CT which was done less than $1 \mathrm{~h}$ after admission showed generalized brain edema and evidence of brain herniation. Also, the duration of cardiac arrest was less than 1 min which would unlikely result in hypoxic brain changes. On the other hand, cardiac arrest is not usual in a single seizure, and cardiac arrest probably was due to brain herniation.

There are few case reports of encephalitis and meningitis associated with COVID-19. These cases presented with fever and flu-like symptoms for few days before manifesting and corpus striatum, and sagittal T2-weighted MR image shows downward herniation of the cerebellar tonsils and brain stem with elongation of the 4 th ventricle

neurological symptoms. The clinical phenotype was mild, and all patients recovered. [1, 3, 4] Brain imaging in COVID-19 encephalitis has shown hyperintensities involving the mesial temporal lobe, gyrus rectus, and olfactory bulb; another patient revealed acute necrotizing hemorrhagic encephalopathy $[4,5]$.

Several CNS manifestations of COVID-19 have been reported including meningitis, encephalitis, stroke, seizures, and encephalopathy. [2] Mechanism by which SARS-CoV-2 causes encephalitis is not completely understood.

\section{Limitation}

We were not able to perform CSF examination in our case due to raised ICT and hence cannot confirm the presence of SARS COV-2 in CSF or intrathecal synthesis of immunoglobulins. But the clinical presentation and brain imaging were suggestive of fulminant encephalitis.

\section{Conclusions}

As SARS COV-2 is a neurotropic virus, neurological manifestations are not uncommon. Neurological manifestations can range from mild agitation to severe encephalitis. This case highlights the fulminant encephalitis as one extreme of the spectrum of CNS manifestation in COVID-19.

Acknowledgments We acknowledge Dr. Saeid Haghighi who survived life of thousands of patients and had active contribution during COVID19 pandemic and lost his life due to COVID-19. Also, we acknowledge his family which gave us permission to write up his medical history.

Authors' contribution 1) Research project: A. conception, B. organization, C. execution

2)Statistical analysis: A. design, B. execution, C. review and critique 3 Manuscript: A. writing of the first draft, B. review and critique. ME: 1A, 1B, 1C, 3B 
MS: 1A, 1B, 1C, 2A, 2B, 2C, 3A

AAM: $1 \mathrm{C}, 2 \mathrm{C}, 3 \mathrm{C}$

SHA: $1 \mathrm{C}, 3 \mathrm{~B}$

\section{Compliance with ethical standards}

Conflict of interest The authors declare that they have no conflict of interest.

Ethical approval None.

\section{References}

1. Moriguchi T, Harii N, Goto J, Harada D, Sugawara H, Takaminoa J, Uenoa M, Sakataa H, Kondoa K, Myosea N, Nakaoc A, Takedad M, Haroe H, Inouef O, Suzuki-Inoueg K, Kubokawah K, Ogiharai S, Sasakig T, Kinouchij H, Hiroyuki Kojin T (2020) A first case of meningitis/encephalitis associated with SARS-Coronavirus-2. Int J 94(January):55-58. https://doi.org/10.1016/j.ijid.2020.03.062
2. Rogers JP, Chesney E, Oliver D, Pollak TA, McGuire P, Fusar-Poli P, Zandi MS, Lewis G, David AS (May 2020) Psychiatric and neuropsychiatric presentations associated with severe coronavirus infections: a systematic review and meta-analysis with comparison to the COVID-19 pandemic. Lancet Psychiatry 7:611-627. https://doi.org/ 10.1016/S2215-0366(20)30203-0

3. Bernard-Valnet R, Pizzarotti B, Anichini A, Demars Y, Russo E, Schmidhauser M, Cerutti-Sola J, Rossetti AO, du Pasquier R (2020) Two patients with acute meningo-encephalitis concomitant to SARS-CoV-2 infection. Eur J Neurol 27:0-1. https://doi.org/10. 1111/ene. 14298

4. Montalvan V, Lee J, Bueso T, De Toledo J, Rivas K (2020) Neurological manifestations of COVID-19 and other coronavirus infections: a systematic review. J Neurol Sci 194(January). https:// doi.org/10.1016/j.clineuro.2020.105921

5. Politi LS, Salsano E, Grimaldi M (2020) Magnetic Resonance Imaging Alteration oft he Brain in a Patient with Coronavirus Disease 2019 (COVID-19) and Anosmia. JAMA Neurol. https:// doi.org/10.1002/jmv.25824

Publisher's note Springer Nature remains neutral with regard to jurisdictional claims in published maps and institutional affiliations. 